\title{
FACTORES DE RIESGO DEPENDIENTES DEL HUÉSPED PARA NEUMONÍA ASOCIADA A VENTILADOR EN LA UCI DEL HOSPITAL NACIONAL DOS DE MAYO ENERO 2013 - ENERO 2015
}

\author{
RISK FACTOR DEPENDENT ON THE HOST FOR VAP IN THE ICU OF THE NATIONAL \\ HOSPITAL DOS DE MAYO JANUARY 2013 - JANUARY 2015
}

Katherine Luisa Contreras-Gala', Edwin Castillo-Velarde'1, Jhony A. De La Cruz-Vargas', Patricia Segura-Nuñez¹

\begin{abstract}
RESUMEN
Objetivos: Evaluar la asociación entre los factores de riesgo dependientes del huésped y la neumonía asociada a ventilador en la UCl del Hospital Nacional Dos de Mayo durante enero 2013 a enero 2015. Métodos: Estudio observacional, cuantitativo, analítico y retrospectivo. Se estudió factores de riesgo dependientes del huésped para Neumonía Asociada a Ventilador (NAV) y se evaluó la asociación de cada uno de ellos con la enfermedad de estudio. En el análisis de resultados, se utilizó los Odds ratios (OR) crudos y el análisis bivariado. Resultados: Durante el periodo de estudio, 232 pacientes ventilados fueron evaluados, de los cuales fueron diagnosticados con NAV, 18 pacientes (7.75\%), siendo la tasa de letalidad de $27.77 \%$. El sexo masculino y la edad avanzada mostraron un OR de 0.846 (IC 95\% 0.322 -2.227) y 1.017 (IC 95\% 0.386- 2.677), respectivamente. En relación a EPID, EPOC, neumotórax y tuberculosis, se obtuvieron OR de 1.739 (IC 95\% 0.202-14.976), 3.619 (IC 95\% 1.30610.031), 1.6332 (IC 95\% 0.0812 - 32.8400) y 2.2973 (IC 95\% 0.1063 - 49.6550), respectivamente. En relación a neoplasias, Diabetes Mellitus, VIH y lupus eritematoso sistémico, se encontraron OR de 0.5264 (IC $95 \% 0.0296$ - 9.3464), 0.8669 (IC 95\% 0.0470 - 16.0025), 15.000 (IC 95\% 3.388 - 66.407) y 26.625 (IC 95\% $2.289-309.663$ ), respectivamente. En relación al sobrepeso, se encontró un OR de 10.500 (IC 95\% 2.150 - 51.281). Conclusión: El sexo masculino y la edad avanzada mostraron una tendencia a ser factores de riesgo para NAV, sin alcanzar significancia estadística. EI EPOC, VIH, LES y el sobrepeso fueron los factores que sí mostraron una asociación estadísticamente significativa con NAV.
\end{abstract}

Palabras clave: Neumonía; Ventilador; Estancia hospitalaria; UCI. (fuente: DeCS BIREME)

\begin{abstract}
Objective: To evaluate the association between host-dependent risk factors and ventilator-associated pneumonia in the ICU of the Hospital Nacional Dos de Mayo during January 2013 to January 2015. Methods: Observational, quantitative, analytical and retrospective study. Host-dependent risk factors for Ventilator Associated Pneumonia (VAP) were studied and the association of each of them with the disease studied was evaluated. In the analysis of results, crude odds ratios (OR) and bivariate analysis were used. Results: During the study period, 232 patients were evaluated, of which 18 patients $(7.75 \%)$ were diagnosed with VAP; the mortality rate was $27.77 \%$. Males and advanced age showed an OR of $0.846(95 \% \mathrm{Cl} 0.322-2.227)$ and 1.017 (95\% Cl 0.386-2.677), respectively. In relation to EPID, EPOC, pneumothorax and tuberculosis, ORs were obtained from 1,739 (95\% Cl 0.202-14.976), 3.619 (95\% Cl 1.306-10.031), 1.6332 (95\% Cl $0.0812-32.8400$ ) and 2.2973 (CI $95 \% 0.1063$ - 49.6550), respectively. In relation to neoplasms, Diabetes Mellitus, HIV and systemic lupus erythematous, ORs were found of 0.5264 (95\% Cl $0.0296-9.3464), 0.8669$ (Cl 95\% $0.0470-16.0025$ ), 15.000 (Cl 95\% 3.388 - 66.407) and 26.625 (95\% Cl 2.289-309.663), respectively. In relation to overweight, an OR of 10,500 was found $(95 \% \mathrm{Cl} 2,150-51,281)$. Conclusions: Male sex and advanced age showed a tendency to be risk factors for VAP, without reaching statistical significance. COPD, HIV, SLE and overweight were the factors that did show a statistically significant association with NAV.
\end{abstract}

Key words: Pneumonia; Ventilator; Hospital stay; ICU. (source: MeSH NLM)

\footnotetext{
1 Instituto de Investigación en Ciencias Biomédicas (INICIB), Facultad de Medicina Humana, Universidad Ricardo Palma (FAMURP). Lima, Perú. Correspondencia: Katherine Luisa Contreras-Gala. Dirección: Jr. Trinidad Moráb 275. Urbanización El Retablo I etapa. Comas. Celular: 979483818 Correo: katyc19@hotmail.com
}

Citar como: Katherine Luisa Contreras-Gala, Edwin Castillo-Velarde, Jhony A. De La Cruz-Vargas, Patricia Segura-Nuñez. Factores de riesgo dependientes del huésped para neumonía asociada a ventilador en la UCl del Hospital Nacional Dos de mayo, enero 2013 - enero 2015 [Artículo Original]. Rev. Fac. Med. Hum. 2016;16(3):60-68. DOI 10.25176/RFMH.v16.n3.654 


\section{INTRODUCCIÓN}

La neumonía intrahospitalaria ocupa un punto de atención especial en las unidades de cuidados intensivos ( $\mathrm{UCl}$ ) ya que representa la primera causa de infección más común adquirida en ellas ${ }^{3}$, y su riesgo se ve incrementado más de 20 veces por la presencia de la vía aérea artificial ${ }^{4}$.

En nuestro país, según el MINSA, en el último estudio de prioridades nacionales de investigación en salud 2015-2021, las neumonías se encuentran se encuentran como primer lugar en los problemas sanitarios que deben priorizarse en el Perú'.

La Neumonía asociada a Ventilador constituye una de las causas más comunes de infección nosocomial en la unidad de cuidados intensivo $s^{8}$ y la Unidad de Cuidados Intensivos del Hospital Nacional Dos de Mayo (nivel III$1)^{9}$ no es la excepción. En un estudio longitudinal de casos y controles en el 2011, realizado en la unidad de cuidados intensivos del Hospital Nacional Dos de Mayo se describe una incidencia de neumonía intrahospitalaria del $11 \%{ }^{3}$. De todos los pacientes con neumonía intrahospitalaria evaluados en este estudio, el 100\% tuvieron ventilación mecánica ${ }^{3}$.

Al día de hoy, se conoce que la neumonía asociada a ventilador se encuentra relacionada a múltiples factores de riesgo, dentro de los cuales tenemos a los factores extrínsecos o de intervención, entre los cuales podemos mencionar a la duración de la ventilación mecánica, el cambio frecuente en el circuito del ventilador, el tipo de circuito, el número de reintubaciones, el uso de agentes de sedación, el uso de antihistamínicos y el transporte fuera de la UCl; y a los factores intrínsecos o dependientes del huésped5, como por ejemplo el sexo masculino, la edad avanzada, el sobrepeso, EPID, EPOC, enfermedades inmunosupresoras como diabetes mellitus, etcétera. Según la literatura revisada, se puede estimar que los factores de riesgo extrínsecos asociados a NAV corresponden aproximadamente al $80-85 \%$, mientras que los factores intrínsecos corresponderían sólo a un 15-20\%³.

Hoy en día, a pesar de que existen estudios que evalúan en general, los factores de riesgo asociados a NAV, existe carencia de estudios relacionados específicamente a los factores dependientes del huésped y su asociación con el desarrollo de una NAV.
Con el propósito de adquirir un mayor conocimiento acerca de dicha asociación, de tal forma que podamos orientar mejor la instauración de medidas preventivas en este grupo de riesgo, se llevó a cabo el presente trabajo de investigación, el cual tuvo como objetivo principal evaluar la asociación entre los factores de riesgo dependientes del huésped y la neumonía asociada a ventilador en la UCl del Hospital Nacional Dos de Mayo durante enero 2013 a enero 2015.

\section{MÉTODOS}

Se realizó un estudio de diseño observacional, cuantitativo, analítico, y retrospectivo. Se evaluó a todos los pacientes que fueron hospitalizados $y$ que hayan requerido ventilación mecánica en la UCl durante el periodo Enero 2013 a Enero 2015, siguiendo las pautas de nuestros criterios de inclusión y exclusión.

Se estudió a toda la población y se evaluó la presencia de factores de riesgo dependientes de huésped en los pacientes afectados con NAV (edad avanzada, sexo masculino, enfermedades respiratorias previas, enfermedades inmunosupresoras y sobrepeso/ obesidad) y la asociación existente entre estos factores y la enfermedad en estudio. En relación al estado nutricional, se utilizó la clasificación del IMC de la Organización Mundial de la Salud (OMS) ${ }^{28}$.

Los cálculos estadísticos se hicieron utilizando el programa SPSS 22.0 y para la elaboración de tablas, diagramas y gráficos, se utilizó el programa Microsoft Excel versión 2013.

\section{RESULTADOS}

Durante el periodo de estudio Enero 2013 a Enero 2015 en la Unidad de Cuidados Intensivos del Hospital Nacional Dos de Mayo, 232 pacientes con ventilación mecánica fueron evaluados, de los cuales fueron diagnosticados con NAV, 18 pacientes (7.75\%), siendo la tasa de letalidad en el presente estudio de $27.77 \%$ (Tabla N01 y Gráfico N01). 
Tabla 1. Características demográficas, epidemilógicas y clínicas de 232 pacientes admitidos en la Unidad de Cuidados Intensivos del Hospital Nacional Dos de Mayo de enero 2013 a enero 2015.

\section{Caracteristicas}

frecuencia

Porcentaje válido (\%)

\section{Características Demográficas}

$\mathrm{N}=232$

NAV

18

6

12

214

NO NAV

Caracteristicas epidemilógicas

Sexo

Sexo Masculino

Sexo Femenino

Edad

Edad $\geq 60$ años

128

104

Edad $<60$ años

Caracteristicas clínicas

EPID

EPID

No EPID

EPOC

EPOC

No EPOC

39

193

Neumotórax

Neumotórax

No Neumotórax

3

229

1.3

98.7

Tuberculosis

Tuberculosis

No Tuberculosis

230

99.1

\section{Neoplasias}

Neoplasias

No Neoplasias

10

4.3

222

95.7

VIH

VIH

No VIH

224 


\section{Diabetes Mellitus}

Diabetes Mellitus

$$
6
$$

No Diabetes Mellitus

226

97.4

Lupus eritematoso sistémico

Lupus Eritematoso Sistémico 3

No Lupus Eritematoso Sistémico

229

98.7

\section{Sobrepeso / Obesidad}

Sobrepeso / Obesidad 7

No Sobrepeso / Obesidad

225

97

\begin{tabular}{lcc} 
Indicadores de mortalidad & & \\
Letalidad de Nav & 5 & 27.77 \\
\hline Muertos & 13 & 72.23 \\
\hline Vivos & 18 & \\
Total pacientes con Nav & & 38.36 \\
Mortalidad global & 89 & 61.64 \\
Vivos & 143 & 100.00 \\
\hline Total pacientes & 232 & \\
\hline
\end{tabular}

Fuente: Ficha de recolección de datos.

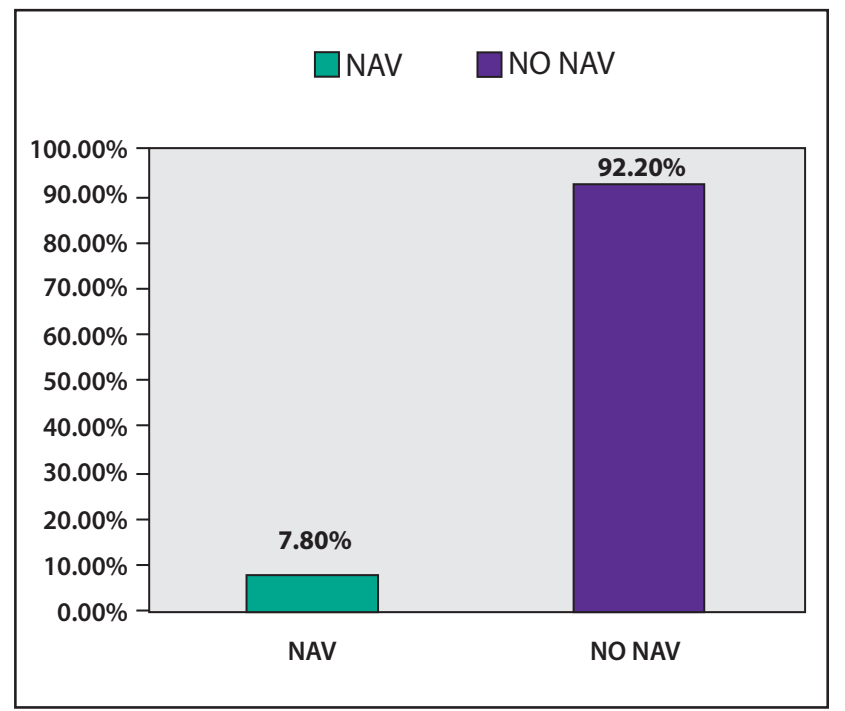

Gráfico 1. Frecuencia de neumonía asociada a ventilador en la UCl del Hospital Nacional Dos de Mayo durante enero 2013 a enero 2015.

Fuente: Ficha de recolección de datos.

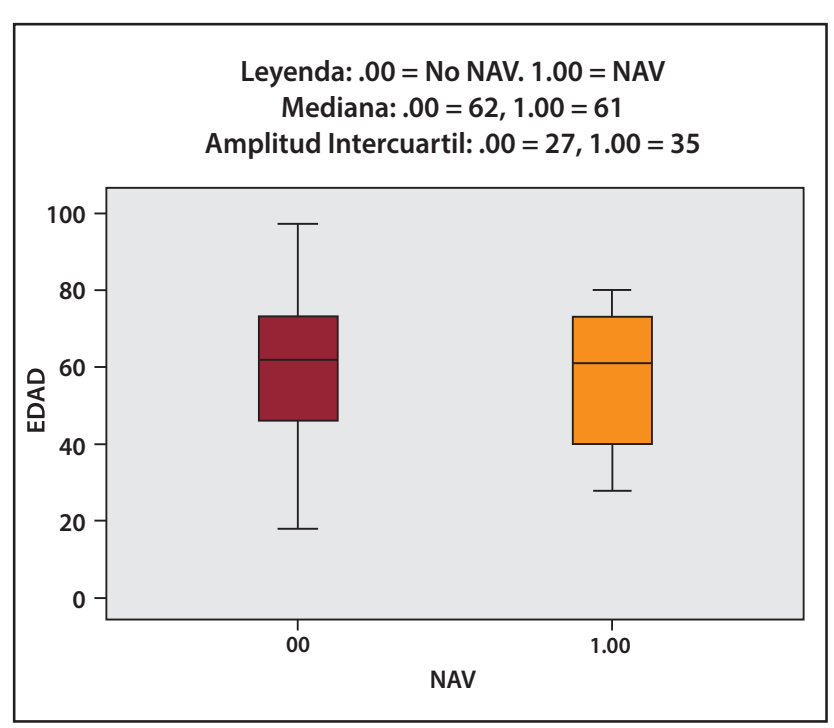

Gráfico 2. Diagrama de cajas de la variable Neumonía Asociada a ventilador en relación a edad en la $\mathrm{UCl}$ del Hospital Dos de Mayo durante enero 2013 a enero 2015. Fuente: Ficha de recolección de datos. 
En relación al sexo masculino y su asociación con NAV, se encontró un odds ratio de 0.846 (IC 95\% 0.322 -2.227). La edad avanzada ( $\geq 60$ años) mostró un odds ratio de 1.017 (IC 95\% 0.386- 2.677) (Tabla Nº2).

En el Gráfico N02, el cual corresponde a un diagrama de cajas, se puede observar que en el grupo de pacientes con Neumonía Asociada a Ventilador, la mediana de edad (valor=61) es ligeramente menor que en el grupo de pacientes sin la enfermedad en estudio (Gráfico $\mathrm{N}^{\circ} 02$ ). No obstante, la amplitud Intercuartil que nos permite comparar ambos grupos, brindándonos una media de la extensión representada por la mitad de cada grupo, nos muestra que hay mayor variabilidad de edad en el grupo de pacientes con NAV, siendo la amplitud Intercuartil mayor a la encontrada en el grupo de pacientes sin la enfermedad (35 vs 27) (Gráfico N02).

Tabla 2. Estimación de Odds Ratios de los factores de riesgo dependientes del húesped asociados a la prevalencia de Nav, evaluados en 232 pacientes admitidos en la unidad de cuidados intensivos del Hospital Nacional Dos de Mayo de enero 2013 a enero 2015.

\begin{tabular}{|c|c|c|c|c|}
\hline \multirow{3}{*}{ Factor de riesgo } & \multicolumn{3}{|c|}{ Estimación de Odds Ratios } & \multirow{3}{*}{ Valor de $p$} \\
\hline & & Intervalc & $\mathrm{za}$ al $95 \%$ & \\
\hline & & Inferior & Superior & \\
\hline Sexo masculino & 0.846 & 0.322 & 2.227 & 0.735 \\
\hline Edad avanzada $(\geq 60)$ & 1.017 & 0.386 & 2.677 & 0.973 \\
\hline $\begin{array}{l}\text { Enfermedades } \\
\text { Respiratorias Previas }\end{array}$ & 3.376 & 1.254 & 9.085 & 0.012 \\
\hline EPID & 1.739 & 0.202 & 14.976 & 0.610 \\
\hline EPOC & 3.619 & 1.306 & 10.031 & 0.009 \\
\hline Neumotórax & 1.6332 & 0.0812 & 32.8400 & 0.7487 \\
\hline Tuberculosis & 2.2973 & 0.1063 & 49.6550 & 0.5958 \\
\hline $\begin{array}{l}\text { Enfermedades } \\
\text { Inmunosupresoras }\end{array}$ & 4.595 & 1.563 & 13.511 & 0.003 \\
\hline Neoplasias & 0.5264 & 0.0296 & 9.3464 & 0.6619 \\
\hline VIH & 15.000 & 3.388 & 66.407 & 0.000 \\
\hline Diabetes Mellitus & 0.8669 & 0.0470 & 16.0025 & 0.9235 \\
\hline LES & 26.625 & 2.289 & 309.663 & 0.000 \\
\hline Sobrepeso & 10.500 & 2.150 & 51.281 & 0.000 \\
\hline
\end{tabular}


Tabla 3. Odds Ratios significativos de los factores de riesgos dependientes del huésped asociados a la prevalencia de NAV, evaluados en 232 pacientes admitidos en la unidad de cuidados intensivos del Hospital Dos de Mayo de enero 2013 a enero 2015.

\section{Estimación de Odds Ratios}

Intervalo de Confianza al 95\%

$\begin{array}{llll}\text { Factor de riesgo } & \text { OR } & \text { Valor de } \mathrm{p}\end{array}$

$\begin{array}{llll}\text { LES } & 26.625 & 2.289 & 309.663\end{array}$

\begin{tabular}{lcccc}
$\mathrm{VIH}$ & 15.000 & 3.388 & 66.407 & 0.000 \\
$\begin{array}{l}\text { Sobrepeso } \\
\text { Enfermedades } \\
\text { inmunosupresoras }\end{array}$ & 10.500 & 2.150 & 51.281 & 0.000 \\
\hline EPOC & 4.595 & 1.563 & 13.511 & 0.003 \\
\hline $\begin{array}{l}\text { Enfermedades } \\
\text { Respiratorias Previas }\end{array}$ & 3.619 & 1.306 & 10.031 & 0.009 \\
\hline
\end{tabular}

Fuente: Ficha de recolección de datos.

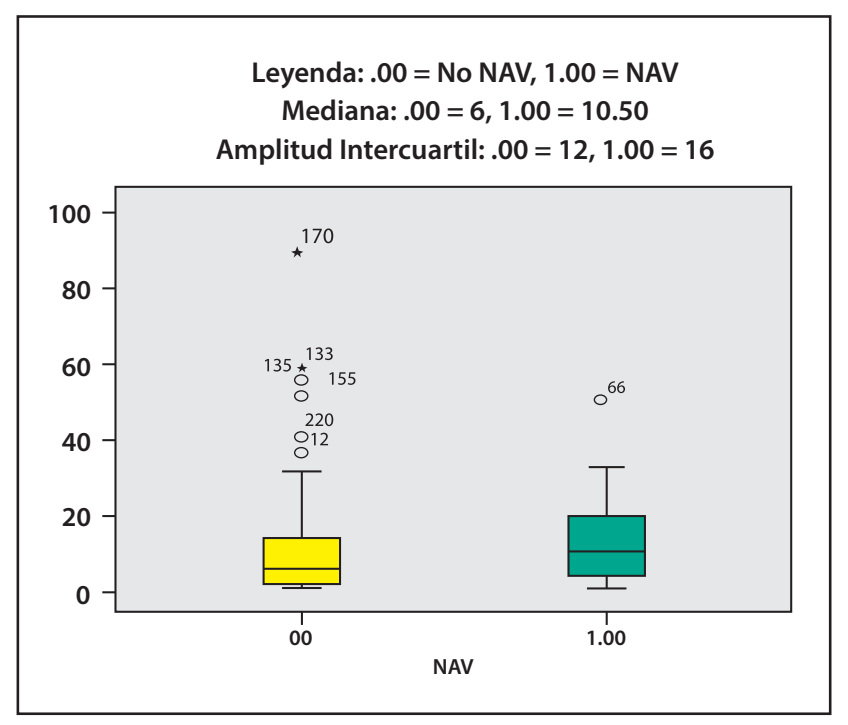

Gráfico 3. Diagrama de cajas de la variable Neumonía Asociada a ventilador en relación a Estancia Hospitalaria en la UCl del Hospital Nacional Dos de Mayo durante enero 2013 a enero 2015.

Fuente: Ficha de recolección de datos.

El gráfico $\mathrm{N}^{\circ} 03$ nos muestra que la mediana de estancia hospitalaria en pacientes de la UCI del Hospital Nacional

Dos de Mayo durante enero 2013 a enero 2015, fue mayor en los pacientes con NAV (10.50 días vs 6 días) (Gráfico N03).
En relación a las enfermedades respiratorias previas, se evaluó primero a todas estas en su conjunto (EPID, EPOC, neumotórax y tuberculosis), y se encontró un Odds Ratio de 3.376 (IC 95\% 1.254- 9.085). Luego se evaluó a cada factor por separado, encontrándose en relación a EPID y EPOC, valores de odds ratios de 1.739 (IC 95\% 0.202-14.976) y 3.619 (IC 95\% 1.306-10.031) respectivamente (Tabla $N^{\circ} 02$, Gráfico $N^{\circ} 04$ ). En relación a Neumotórax y Tuberculosis, se obtuvo Odds Ratio de 1.6332 (IC 95\% 0.0812 - 32.8400) y de 2.2973 (IC 95\% $0.1063-49.6550)$ respectivamente (Tabla $\mathrm{N}^{\circ} 02 \mathrm{y} \mathrm{N}^{\circ} 03$ ).

En relación a las enfermedades inmunosupresoras, de manera similar a cómo se procedió con las enfermedades respiratorias previas, se evaluó primero a todas estas enfermedades en su conjunto (Neoplasias, VIH, Diabetes Mellitus y Lupus Eritematoso Sistémico), y se obtuvo un Odds Ratio de 4.595 (IC 95\% 1.563 -13.511) (Tabla N02 y N03). Tras la evaluación individual de cada factor, en relación a VIH y lupus eritematoso sistémico, se encontró un odds ratio de 15.000 (IC 95\% 3.388 - 66.407) y 26.625 (IC 95\% 2.289 -309.663) respectivamente (Tabla Nº2). 


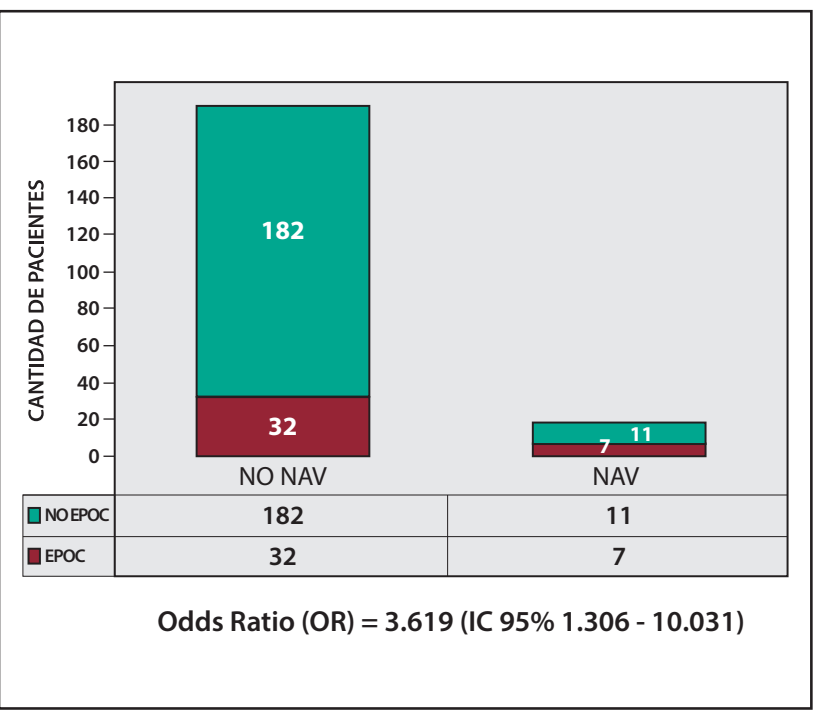

Gráfico 4. Distribución de 232 pacientes evaluados en la UCI del Hospital Nacional Dos de Mayo según EPOC y la presencia de NAV durante enero 2013 a enero 2015. Fuente: Ficha de recolección de datos.

En relación a Diabetes Mellitus y Neoplasias, se obtuvo Odds Ratios de 0.8669 (IC 95\% 0.0470 - 16.0025) y de 0.5264 (IC 95\% 0.0296 - 9.3464) respectivamente (Tabla N02).

En relación al último objetivo planteado de la presente investigación, el cual busca verificar la asociación entre sobrepeso/obesidad y NAV, se encontró de forma interesante para el sobrepeso, un OR de 10.500 (IC 95\% 2.150 - 51.281) (Tabla N03). No se encontró pacientes con IMC correspondiente a obesidad. En el presente estudio, no se realizó el análisis multivariado con subgrupos, debido al bajo número de casos de pacientes con NAV, lo cual afectaría el poder estadístico.

\section{DISCUSIÓN}

La prevalencia de NAV en la población estudiada fue del $7.75 \%$, lo cual se encuentra cercano a lo encontrado por Otiniano Loyola A et al en la UCl del Hospital Daniel Alcides Carrión durante enero-diciembre 2010, en el cual se encontró una prevalencia del $11 \%^{3}$. Lo encontrado en nuestro estudio difiere de lo hallado por Neiva Acosta et al en la UCl del Hospital Departamental de Villavicencio-Colombia, en el periodo Junio 2007 a Junio 2008, donde la NAV mostró una frecuencia de $19 \%{ }^{23}$ y de lo encontrado por Xie Duo-shuang et al, quien encuentra una frecuencia de NAV de $20.9 \%{ }^{24}$. Esta disimilitud se deba probablemente a las diferentes realidades tanto a nivel socioeconómico, así como también en el ámbito de accesibilidad a los servicios de salud.
En el presente estudio, la tasa de letalidad asociada a NAV fue $27.77 \%$. Esto difiere de lo encontrado por Montalvo et al en el Hospital Dos de Mayo durante enero 2006 a diciembre 2010, quien encontró una tasa de letalidad de neumonía nosocomial de $36,2 \%{ }^{27}$; de lo encontrado por Pawar et al, en el 2003, en Nueva Delhi-India, donde se reportó una tasa de mortalidad asociada a NAV del $16 \%$ en comparación con un $0.2 \%$ en casos de pacientes que no presentaron NAV20; y de lo encontrado por Delle Rose et al de septiembre 2010 a septiembre 2011 , donde se obtuvo una tasa de letalidad del $44,3 \%{ }^{29}$.

En relación al sexo masculino, se encontró un odds ratio de 0.846 (IC 95\% $0.322-2.227$ ), En relación al sexo masculino y su asociación con NAV, se encontró un odds ratio de 0.846 (IC 95\% 0.322 -2.227), el cual al ser un valor muy cercano al neutro, nos permite inferir que es un factor con tendencia a ser un factor de riesgo para NAV, pero sin llegar a alcanzar significancia estadística.

Lo encontrado en nuestro estudio difiere de lo hallado por otros autores, y entre ellos, destaca el estudio realizado por Xie Duo-shuang et al 24, donde se encuentra una asociación estadísticamente significativa.

La edad avanzada ( $\geq 60$ años) mostró un odds ratio de 1.017 (IC 95\% 0.386- 2.677), lo cual nos permite afirmar que la edad avanzada tiene tendencia a ser un factor de riesgo para NAV, pero esta asociación no alcanza significancia estadística en el presente estudio. Lo hallado concuerda con lo encontrado por Neiva Acosta et al. ${ }^{23}$; no obstante difiere a lo encontrado por Badawy M.Sh et al. ${ }^{12}$.

En relación a las enfermedades respiratorias previas, se evaluó primero a todas estas en su conjunto (EPID, EPOC, neumotórax y tuberculosis), y se encontró un Odds Ratio de 3.376 (IC 95\% 1.254- 9.085), lo cual evidenció una asociación estadísticamente significativa. Lo encontrado en nuestro estudio concuerda con lo encontrado por Giard $\mathrm{M}$ et al, quien concluye que la aparición de neumonía asociada a ventilador tardía depende mucho de enfermedades anteriores, destacando traumatismos y enfermedades respiratorias previas ${ }^{22}$.

De todas las enfermedades respiratorias previas estudiadas, la EPOC mostró ser un factor de riesgo significativo para NAV, y esto se puede deber probablemente a la inhibición de la función mucociliar, a la presencia de daño pulmonar estructural, y probablemente también al uso excesivo 
de corticosteroides en su manejo, lo cual ocasionaría también un estado de inmunosupresión en estos pacientes.

En relación a las enfermedades inmunosupresoras, de manera similar a cómo se procedió con las enfermedades respiratorias previas, se evaluó primero a todas estas enfermedades en su conjunto y tras la evaluación individual de cada factor, se encontró que tanto el VIH como el LES mostraron la mayor significancia estadística para ser factores de riesgo para NAV en la presente investigación. No obstante, estos resultados deben tomarse con cautela debido a que la heterogeneidad entre los grupos de los pacientes con la enfermedad y de los pacientes sin la enfermedad, constituye de por sí un sesgo de interpretación para las asociaciones descritas. La asociación encontrada entre estos factores y NAV, podría explicarse por el hecho de que cualquier enfermedad subyacente que altere de por sí gravemente el estado inmunológico del paciente, consecuentemente, va a agravar también la evolución de cualquier infección que pueda surgir durante la estancia hospitalaria.

De acuerdo a los resultados de odds ratios obtenidos, se puede afirmar que la diabetes mellitus y las neoplasias sólo presentan una tendencia de asociación con NAV, mas no llegan a un rango de significancia estadística.

Se encontró de forma interesante para el sobrepeso, un OR de 10.500 (IC 95\% 2.150 - 51.281), resultado que evidencia que el factor sobrepeso es un factor de riesgo estadísticamente significativo para la enfermedad en estudio, en la presente investigación. Este hallazgo concuerda con lo encontrado por Delle Rose et al. ${ }^{29}$.

Lo encontrado en relación a la asociación entre sobrepeso y NAV, se presume que puede deberse al hecho de que el sobrepeso y la obesidad muy probablemente alterarían la mecánica ventilatoria, resultando finalmente en un incremento del trabajo respiratorio y dificultad en el intercambio gaseoso. No obstante, luego de una búsqueda exhaustiva, no se encuentra bibliografía en la cual se señale y explique claramente las razones de la asociación encontrada entre obesidad e infección.

\section{CONCLUSIÓN}

En base a los resultados, se puede concluir que en la población estudiada, dentro de los factores dependientes del huésped o intrínsecos de NAV, el sexo masculino y la edad avanzada no mostraron tendencia a ser un factor de riesgo para NAV.

Dentro de las enfermedades respiratorias previas, la EPOC fue el factor que mostró mayor tendencia a ser un factor de riesgo para NAV, obteniéndose significancia estadística en dicha asociación.

De las enfermedades inmunosupresoras y en general, en el presente estudio, el LES y el VIH mostraron la mayor tendencia a ser factores de riesgo de NAV, respectivamente, evidenciando los Odds Ratios más altos y alcanzando significancia estadística en dichas asociaciones.

Finalmente, el sobrepeso mostró una fuerte tendencia a ser un factor de riesgo para NAV, evidenciando significancia estadística en la presente investigación.

\section{RECOMENDACIÓN}

Si bien el presente trabajo de investigación encuentra resultados importantes, es relevante mencionar que sólo evaluamos factores de riesgo intrínsecos que se asocian a la patología en estudio, lo cual aproximadamente sólo representa un 15-20\% de todos los factores asociados a NAV. Por este motivo, recomendamos dar continuidad al análisis de los otros factores asociados a NAV de una manera prospectiva para mayor enriquecimiento de los conocimientos de la profesión médica en cuanto a los factores influyentes en la presencia del evento. Asimismo, sería importante aumentar la población de estudio para obtener un mayor poder estadístico.

Financiamiento: Autofinanciado.

Conflicto de interés: Los autores declaran no tener conflictos de interés en la publicación de este artículo.

Recibido: 09 de diciembre

Aprobado: 28 de diciembre 


\section{REFERENCIAS BIBLIOGRÁFICAS}

1. Ministerio de Salud. Prioridades Nacionales de Investigación en Salud 2015-2021. Lima: Minsa; 2015.

2. Universidad Ricardo Palma-Facultad de Medicina Humana-Instituto de Investigación en Ciencias Biomédicas: INICIB.Prioridades de Investigación en Salud 2016-2021. Lima; Febrero 2016.

3. Otiniano Loyola A, Gómez Arce M. Factores de riesgo asociados a neumonía intrahospitalaria en pacientes de la unidad de cuidados intensivos. Rev Soc Peru Med Interna 2011; 24 (3): 121-127.

4. Díaz E, Lorente L, Valles J, Rello J. Neumonía asociada a ventilación mecánica. Med. Intensiva 2010; 34(5): 318-324.

5. Charles MVP, Kali A, Easow JM, Joseph NM, Ravishankar M, Srinivasan S

et al. Ventilator-associated pneumonia. AMJ 2014; 7 (8): 334-344.

6. Joseph NM, Sistla S, Dutta TK, Badhe AS, Parija SC. Ventilator-associated pneumonia: a review. European Journal of Internal Medicine 2010; 21 (5) 360-368.

7. Hidalgo LF, Marroquín JE, Antigoni J, Samalvides F. Prevalencia de infecciones hospitalarias en un hospital peruano de nivel IV, en el año 2008. Rev Med Hered 2011; 22 (2): 76-81.

8. Waters B, Muscedere J. A 2015 Update on Ventilator-Associated Pneumonia: New Insights on Its Prevention, Diagnosis, and Treatment. Curr Infect Dis Rep 2015; 17(8): 496.

9. Salaverry O, Cárdenas Rojas D. Establecimientos Asistenciales del Sector Salud, Perú 2009. Rev Peru Med Exp Salud Pública. 2009; 26(2): 264-67.

10. Organización Mundial de la Salud. Prevención de las Infecciones Nosocomiales Guía Práctica (2da edición). [monografías en Internet]* secretaría general de la OMS; 2003 [22/05/16]* disponible en: http://www. who.int/csr/resources/publications/ES_WHO_CDS_CSR_EPH_2002_12. pdf

11. Alvarez Gutierrez FJ. Neumonía nosocomial. Neumosur [Internet]. Sevilla: Asociación de Neumología y Cirugía Torácica del Sur; [actualizado el 17 de noviembre de 2015; acceso 25 de abril de 2016]. Disponible en: http://www.neumosur.net/files/EB03-40\%20nosocomial.pdf

12. Badawy M.Sh, Omar HM, Mohamdien HA, Moktar EA, Deaf EA. Evaluation of risk factors of ventilator associated pneumonia on outcome of acute exacerbation of chronic obstructive pulmonary disease. Egypt. J. Chest Dis. Tuberc. 2015; XXX. XXX-XXX

13. Rello Condomines J. Neumonía asociada a ventilación mecánica. ReMI 2004; 4(10). [fecha de acceso 26 de abril de 2016]. URL disponible en: http://remi.uninet.edu/2004/10/REMIC15.htm

14. Chincha O, Cornelio E, Valverde V, Acevedo M. Infecciones intrahospitalarias asociadas a dispositivos invasivos en unidades de cuidados intensivos de un hospital nacional de Lima, Perú. Rev. Perú. Med. Exp. Salud publica 2013; 30 (4): 616-620

15. Harrison F. Principios de Medicina Interna (18 edición). Madrid: McGraw-Hill Interamericana; 2008.

16. Iribarren BO, Aranda TJ, Dorn HL, Ferrada MM, Ugarte EH, Koscina
MV et al. Factores de riesgo para mortalidad en neumonía asociada a ventilación mecánica. Rev Chil Infect 2009; 26 (3): 227-232.

17. Krawinkel MB. Interacción entre la nutrición y las infecciones a nivel global: una revisión. Ann Nutr Metab 2012; 61(1):39-45.

18. Chaires Gutiérrez R, Palacios Chavarría A, Monares Zepeda E, Poblano Morales M, Aguirre Sánchez J, Franco Granillo J. Neumonía asociada a la ventilación mecánica: el reto del diagnóstico. Rev Asoc Mex Med Crit y Ter Int 2013; 27(2):99-106.

19. Córdova Pluma VH, Peña Santibáñez J, Quintero Beltrán M. Neumonía asociada con ventilador en pacientes de la unidad de cuidados intensivos. Med Int Mex 2011; 27(2):160-167.

20. Pawar M, Mehta Y, Khurana P, Chaudhary A, Kulkarni V, Trehan N Ventilator-Associated Pneumonia: Incidence, Risk Factors, Outcome, and Microbiology. Journal of Cardiothoracic and Vascular Anesthesia 2003; 17(1): $22-28$

21. Hernández Piard M, Rodríguez Martínez Z, González Cobo S, Cruz Dueñas C, Fernández Reyes J. Actualización sobre la neumonía nosocomial asociada a la ventilación mecánica. Rev méd electrón 2005; 27 (4) [fecha de acceso 13 de mayo de 2016]. Disponible en: http://www. revmedicaelectronica.sld.cu/index.php/rme/article/view/222/htm

22. Giard M, Lepape A, Allaouchiche B, Guerin C, Lehot JJ, Robert MO et al. Early- and late-onset ventilator-associated pneumonia acquired in the intensive care unit: comparison of risk factors. J Crit Care 2008; 23 (1): 27-33.

23. Neiva Acosta MI, Gómez Parrado CY, Montaño Contreras SC, Pérez Gutiérrez N, Prieto Franklyn E, Teresa Castro A. Factores relacionados con neumonía asociada a ventilación mecánica en una unidad de cuidados intensivos de la Orinoquia colombiana. Acta Med Colomb 2009; 34(4): 164-168.

24. Duo-shuang X, Xiong W, Lai R, Liu L, Gan X, Wang X et al. Ventilatorassociated pneumonia in intensive care units in Hubei Province, China: a multicentre prospective cohort survey. J Hosp Infect 2011; 78 (4): 284- 8.

25. Labaut Arévalo N, Riera Santiesteban R, Pérez Fuentes IA, Castañeda Carrazana Y. Neumonía asociada a la ventilación mecánica en una unidad de cuidados intensivos. Medisan 2011; 15(12): 1759-1764.

26. Barreda De La Cruz M. Neumonías asociada a la ventilación mecánica: Factores de riesgo en la UCl del Hospital Nacional Carlos Seguin Escobedo Essalud Arequipa 2006. [Tesis doctoral] Arequipa; 2006

27. Montalvo R, Alvarezcano J, Huaroto L, López J, Lam C, Mucha R et al. Factores asociados a mortalidad por neumonía nosocomial en un hospital público de Perú. Rev.Peru.Epidemiol 2013; 17 (2): 1-6.

28. Fandiño A, Giraldo S, Martínez C, Aux C, Espinosa R. Factores asociados con los trastornos de la conducta alimentaria en estudiantes universitarios en Cali, Colombia. Colomb Med 2007; 38: 344-351.

29. Delle Rose D, Pezzotti P, Fortunato E, Sordillo P, Gini S, Boros S et al Clinical predictors and microbiology of ventilator-associated pneumonia in the intensive care unit: a retrospective analysis in six Italian hospitals. Eur J Clin Microbiol Infect Dis. 2016; 1-9. 